\title{
Correlation between intervertebral disc degeneration, paraspinal muscle atrophy, and lumbar facet joints degeneration in patients with lumbar disc herniation
}

\author{
Dong Sun, Peng Liu*, Jie Cheng, Zikun Ma, Jingpei Liu and Tingzheng Qin
}

\begin{abstract}
Background: To assess the correlation between lumbar disc degeneration (LDD), multifidus muscle atrophy (LMA), and facet joints degeneration in patients with L4-L5 lumbar disc herniation (LDH).

Methods: Sixty patients with L4-L5 LDH diagnosed by a $1.5 \mathrm{~T} \mathrm{MRI} \mathrm{scanner} \mathrm{were} \mathrm{enrolled} \mathrm{in} \mathrm{the} \mathrm{study} \mathrm{group} \mathrm{and}$ another 60 patients with non-specific back pain were enrolled in the control group. LDD, LMA, and facet joints degeneration were examined and analyzed independently by two independent orthopedic surgeons using T2weighted images. Wilcoxon test was used for analyzing the difference of LDD and facet joints degeneration between L3-L4 and L5-S1 and difference of LMA between the herniated and control groups. Correlation analysis of the three degeneration grades at the same level was determined by Spearman rank correlation test.

Results: In the herniated group, most LMA at L3-L4 level was grade 1 (42, 70.0\%); grade 2 (33, 55.0\%) at L4-L5 level; and grade $3(27,45.0 \%)$ at L5-S1 level. LMA and LDD grading were significantly different between L3-L4 and L5-S1 levels $(P<0.05)$. In the herniation group, the Spearman value for LDD and LMA grading were $0.352(P<0.01)$ at L3-L4 and $0.036(P>0.05)$ at the L5-S1 level. The differences in LMA between the herniated and control groups at the three levels were significant $(P<0.05)$.

Conclusions: Disc degeneration and multifidus muscles atrophy were positively correlated at the L3-L4 disc level. A lumbar extension muscle strengthening program could be helpful in preventing muscle atrophy and lumbar spinal degeneration.
\end{abstract}

Keywords: Lumbar disc herniation, Disc degeneration, Multifidus muscle atrophy, Facet joints degeneration, Lumbar extension muscle strengthening program

\section{Background}

Lumbar disc herniation (LDH) is one of the most frequent causes of low back pain and sciatic pain in adults. The compression by the protruding disc on the dorsal and/or the ventral nerve roots causes low back pain, leg pain (sciatica), muscle spasm, and restriction of trunk movement [1]. The multifidus muscle-the most medially located back muscle and the largest muscle that spans the lumbosacral junction-serves to maintain the erector posture of the trunk and to abduct and rotate

* Correspondence: 844795606@qq.com

Department of Orthopedics, China-Japan Union Hospital of Jilin University, 126 XianTai Street, Changchun 130000, China the trunk. It is also innervated by the dorsal root of the lumbar spinal nerve [2-4].

Several recent studies have investigated the biomechanics and microstructures of the multifidus muscles in patients with LDH $[5,6]$. An association between multifidus muscle degeneration and chronic low back pain, degenerative disc disease, radiculopathy, and scoliosis has been proposed [7-9]. Muscle atrophy and fatty infiltration to the multifidus muscle following minimally invasive lumbar discectomy was also observed [10]. However, there have only been a few reports on magnetic resonance imaging (MRI) analysis of the paraspinal muscles [11], facet joints, and lumbar disc degeneration 
(LDD) in LDH patients, let alone the correlation study of lumbar degenerative changes between herniated and adjacent segments in patients with L4-L5 disc herniation. We hypothesized that lumbar multifidus atrophy (LMA) is correlated with LDH and that severe atrophy exists in the level innervated by the dorsal root of the herniation compression nerve root.

The present study aimed to compare (by MRI scanning) the differences in LMA, LDD, and facet joints degeneration between the L3-L4 and L5-S1 levels in patients with L4-L5 LDH, and to analyze the correlation between LMA and LDD at the L3-L4 and L5-S1 levels.

\section{Methods}

\section{Study participants}

Between January 2015 and April 2015, 60 patients who had unilateral radiculopathy due to L4-L5 level disc herniation diagnosed by $1.5 \mathrm{~T}$ MRI scanner as well as 60 patients (as the control group) with non-specific back pain at our university hospital were enrolled in our study. Patient characteristics are summarized in Table 1.

The inclusion criteria were: no obvious disc degeneration signs such as disc collapse, endplate Modic and Schmorl changes, and high-intensity zone within the posterior annulus; confirmed L5 nerve root compression by herniated mass during surgery [5]; and aged 20 to 70 years old.

The exclusion criteria were: MRI showed L3-L4 and L5S1 segments disc herniation; lumbar spinal canal stenosis; and history of lumbar surgery, neoplasm, infection, or spinal deformity, such as spondylolisthesis $(>3 \mathrm{~mm})$ or scoliosis $\left(>10^{\circ}\right)$.

\section{Imaging parameters}

MRI of lumbar spine was taken by a $1.5 \mathrm{~T}$ MRI scanner (MAGNETOM $^{\circ}$ Verio, A Tim + Dot System; Siemens, Erlangen, Germany), with the participants in the supine position. And the following scans were performed using the following parameters: sagittal T2-weighted images from T12 to the sacrum (TR/TE 2980/122.6, matrix size $208 \times 320$, time to recovery: $3,000-3,600 \mathrm{~ms}$, time to echo: $87-114 \mathrm{~ms}$, and slice thickness: $4 \mathrm{~mm}$ ); and axial T2-weighted images from T12 to S1 (TR/TE 2980/122.6,

Table 1 Patient characteristics of the disc herniation group and the control group

\begin{tabular}{llll}
\hline Variables & Disc herniation group & Control group & $P$ value \\
\hline Age & $48.97 \pm 8.39$ & $46.08 \pm 7.27$ & $>0.05$ \\
Sex, female & $29(48.3 \%)$ & $31(51.7 \%)$ & - \\
History (months) & $9.15 \pm 5.51$ & - & - \\
VAS & $6.60 \pm 1.33$ & - & - \\
\hline
\end{tabular}

Data is presented as mean \pm standard deviation VAS visual analogue scale/Score matrix size $208 \times 320$, time to recovery: $3,000-3,600 \mathrm{~ms}$, time to echo: $87-114 \mathrm{~ms}$, and slice thickness: $4 \mathrm{~mm}$ ).

\section{Muscle morphometry}

Multifidus muscle cross-sectional area (CSA) was determined at the levels of the L3-L4, L4-L5, and L5-S1 intervertebral discs. Multifidus CSA was measured by the semiquantitative grade system published by Patrick [12] (Fig. 1).

\section{Grading of intervertebral disc degeneration}

The modified system comprises of 8 grades for lumbar disc degeneration [13]. The eight grades represented a progression from normal disc to severe disc degeneration. Grade 1 corresponds to no disc degeneration while Grade 8 corresponds to end-stage degeneration.

\section{Grading of the lumbar facet joints osteoarthritis}

Four grades of osteoarthritis of the facet joints were defined using system published by Pathria [14]: grade 0, normal; grade 1, mild degenerative disease; grade 2, moderate degenerative disease; and grade 3 , severe degenerative disease (Fig. 2).

\section{Image assessment}

Sagittal T2-weighted images were used to analyze disc degeneration as they provided a comprehensive perception of disc structure and good tissue differentiation, and axial T2-weighted images were used to analyze facet joints and paraspinal muscle. Two experienced orthopedic surgeons each graded 360 lumbar levels independently.

\section{Statistical analyses}

The analyses were performed using SPSS software (version 20.0; SPSS IBM; Armonk, NY). Intraobserver reproducibility of analysis results of intervertebral disc, facet joints, and muscle CSA degeneration was assessed using weighted Kappa statistics. Wilcoxon rank sum test was used to compare the differences in intervertebral disc, facet joints, and muscle CSA degeneration grade between L3-L4 and L5-S1 as well as the differences in muscle atrophy between the herniated and control group. Spearman's rho testing was used to analyze the correlation between LDD and LMA at L3-L4 and L5-S1.

\section{Results}

A total of 120 patients were included in this retrospective study. There were 29 females and 31 males in the herniation group with an average age of 48.97 \pm 8.39 years (range from 32 to 65 years). There were 31 females and 29 males in the control group, with an average age of 46.08 years (range from 30 to 64 years). There was no significant difference between the two groups (Table 1). 


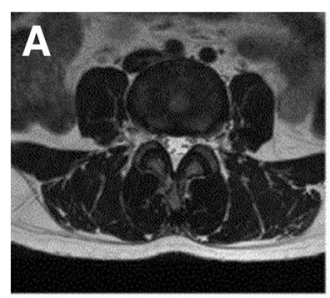

Grade 0

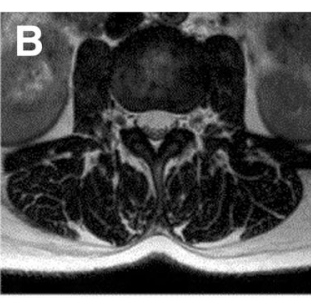

Grade 1

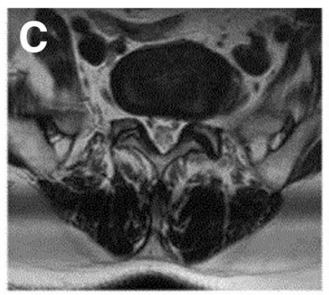

Grade 2

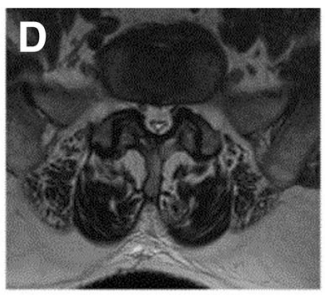

Grade 3

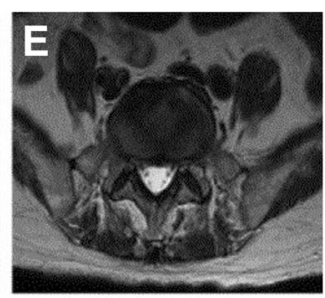

Grade 4

Fig. 1 Goutallier grading (range, 0 to 4) on T1W axial MRls are represented by (a) to (e), respectively [9, 12], and are graded as: grade 0, normal muscle tissue; grade 1, fat streaks; grade 2, more muscle than fat; grade 3, equal amounts of fat and muscle tissue; and grade 4, more fat than muscle

The disc degeneration grading of all patients according to the modified Pfirrmann grading system was shown in Fig. 3. Disc degeneration at the L3-L4 level disc was mostly grade III $(33,55.0 \%)$ and grade IV $(17,28.3 \%)$. L4-L5 disc degeneration was mainly grade V (19, $31.67 \%)$, grade VI $(12,20.0 \%)$, and grade VII $(18,30.0 \%)$. L5-S1 disc degeneration was grade III $(29,48.33 \%)$ and grade IV (17, 28.33\%).

As illustrated in Figs. 3 and 4, according to the Goutallier grading, LMA from the herniated group at the L3-L4 level was mostly grade 1 (42, 70.0\%). LMA at the L4-L5 level was mainly grade $1(22,36.67 \%)$ and grade 2 (33, $55.0 \%)$. L5-S1 level was grade $2(24,40.0 \%)$ and grade $3(27,45.0 \%)$. As for the control group, LMA at the L3-L4 level was grade $0(37,61.67 \%)$ and grade 1 (23, $38.33 \%)$. LMA at L4-L5 and L5-S1 levels were mainly grade 1 (42, 70.0 and 44,73.33\%). There were significant differences in LMA at the three levels between the herniated and control group (Table 2).

The correlation between LDD and LMA at L3-L4, L4L5, and L5-S1 levels is shown in Table 3. Significant correlation was observed at the L3-L4 level (Spearman's rho value $=0.352, P<0.05)$ between LDD and LMA, but no correlation was observed at the L4-L5 and L5-S1 levels.

The Wilcoxon rank sum test result is shown in Table 4. A significant difference in LMA was observed between L3-L4 and L5-S1 levels $(Z=-6.481, P<0.05)$, but no significant difference was found in other levels in LDD and facet joints degeneration (Table 4).

Facet joints degeneration at the L3-L4 level was mainly grade 0 and grade 1 (36.7 and 55.0\%). L4-L5 level facet joints degeneration was mainly grade 1 (61.67\%). Facet joints degeneration at level L5-S1 was mainly grade 0 and 1 (46.67 and 48.33\%) (Fig. 3). The inter-examiner reliability analysis of LDD, LMA, and facets joints degeneration showed excellent result (Kappa $=0.91,0.84,0.86$, and 0.85 , respectively), thus indicating that the measurements were reliable.

\section{Discussion}

In this study, we investigated the correlation between multifidus muscle atrophy, facet joints, and disc degeneration in LDH patients. The result of this study supported our hypothesis that in patients with L4-L5 LDH, multifidus muscle grading was higher at the L5-S1 level than at the L3-L4 level. Positive correlation was identified between LDD and LMA at the L3-L4 level, but no correlation was found at the L5-S1 level.

We found that compared with the control group, herniated patients had more severe LMA at the three levels. There were two possible explanations. First, it could be because the selected patients in the control group had no obvious disc degeneration symptoms. Second, multifidus muscle disuse caused by specific back pain and
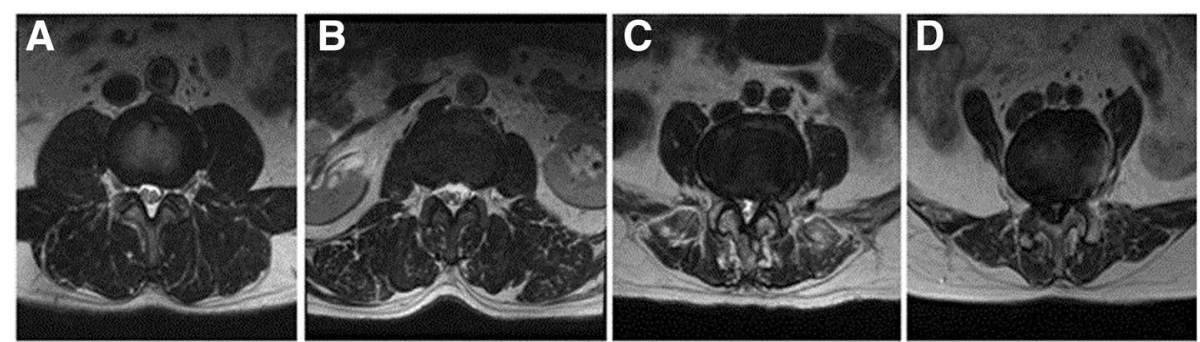

Fig. 2 Examples of MRIs on T2W axial with Pathria grades 0 to 3 are shown [14]. Four grades of osteoarthritis of the facet joints were defined as: (a) grade 0, normal; (b) grade 1, mild degenerative disease; grade 2, moderate degenerative disease; and (c) grade 3, severe degenerative disease 

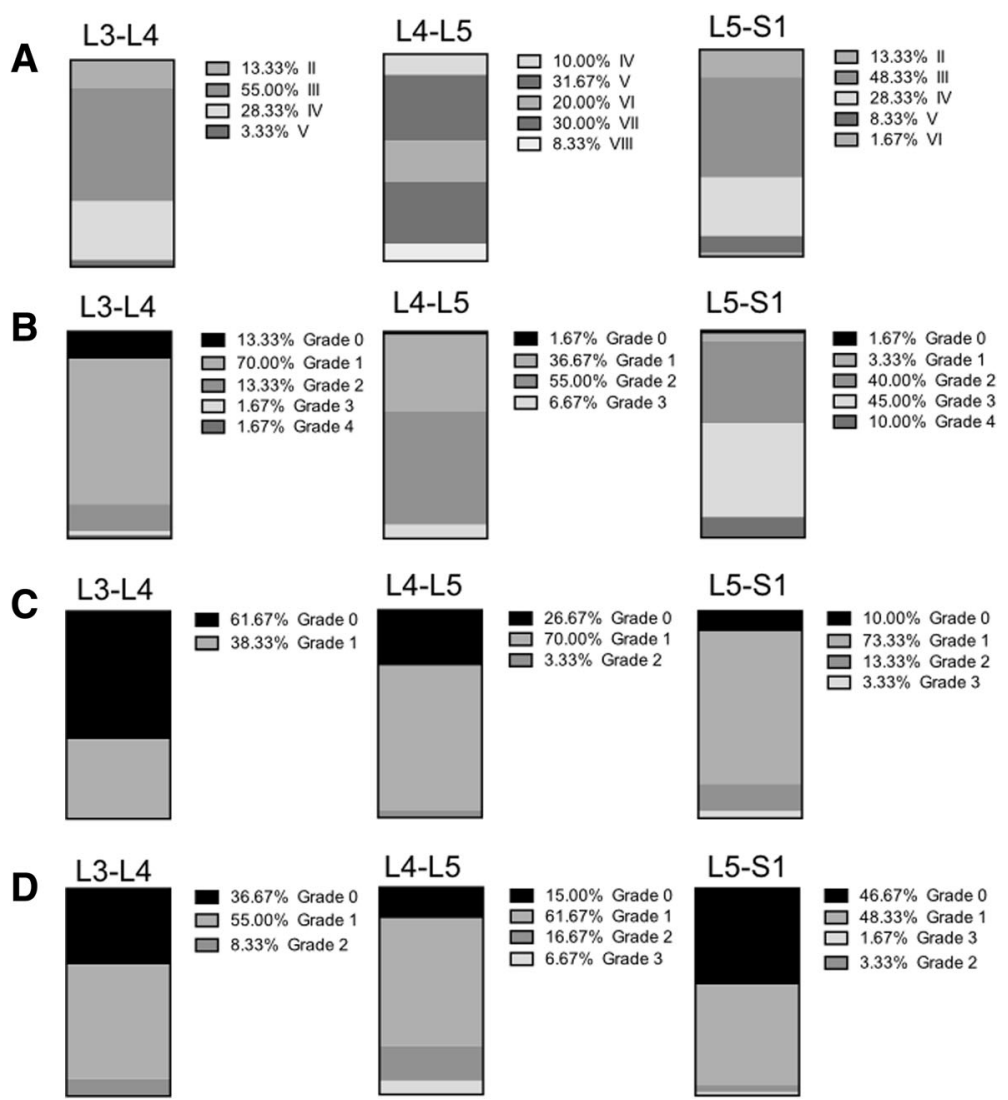

Fig. 3 Lumbar multifidus atrophy, lumbar disc, and facet joints degeneration in the herniated and control groups. a lumbar disc degeneration in the herniated group; $\mathbf{b}$ lumbar multifidus atrophy in the herniated group; c lumbar multifidus atrophy in the control group; $\mathbf{d}$ facet joint degeneration in the herniated group

denervation caused by nerve root compression were the main reasons responsible for this phenomenon.

MRI results from this study demonstrated that there was more severe LMA at the L5-S1 level than at the L3L4 level in L4-L5 LDH patients. Anatomically $[15,16]$, multifidus fascicles arise from the spinous process and adjacent lamina of each lumbar vertebra, descend caudolaterally, traverse L3-L4 vertebral levels, and is innervated by the nerve root of the same level. Similar to the L5 level, multifidus muscle arising from the L5 spinous process is innervated by the L5 nerve root. In patients with L4-L5 LDH, if the L5 root was affected, muscle denervation would be expected only at the L 5 level. The anatomical features result in more muscle volume at the inferior segment than the superior segment.

Two mechanisms for muscle atrophy have been proposed: disuse and denervation. In animal studies $[17,18]$, it is generally agreed that denervation leads to decrease in Type II fiber size. In contrast, opinion differs concerning changes in the Type I fiber size after denervation. However, Yoshihara et al. [19] demonstrated that denervation caused by nerve root compression may lead to atrophy of both fiber types (type I and type II) with structural changes in the human lumbar multifidus muscle.

Despite these controversies, we proposed two mechanisms that are possibly responsible for multifidus atrophy. In LDH patients, disuse/immobilization of the back muscles is common. Such changes may explain the generalized effect (atrophy) at five levels. In addition, symptoms including paraspinal denervation (short-angled fibers) and re-innervation (grouped fibers, polyphasic action potentials) are common in disc herniation and nerve root compression. The CSA of multifidus muscle innervated by medial branch of the dorsal ramus of the L5 nerve root are reduced when L5 nerve root is compressed by herniated mass.

Numerous studies have reported rapid muscle atrophy in response to inflammation $[20,21]$ and muscle or joint injury [19]. However, the mechanisms for such changes are poorly understood. Paraspinal muscle plays a more important role in protecting the L3-L4 segment than others. The multifidus muscle maintains the lumbar lordosis by acting like a bowstring which helps to transmit some of 
Herniated L3-4

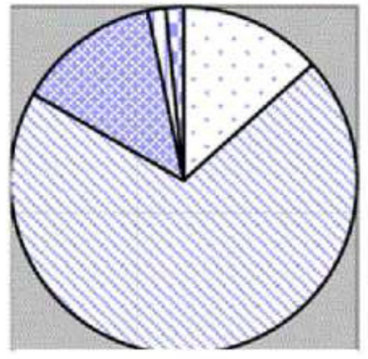

Herniated L5-S1

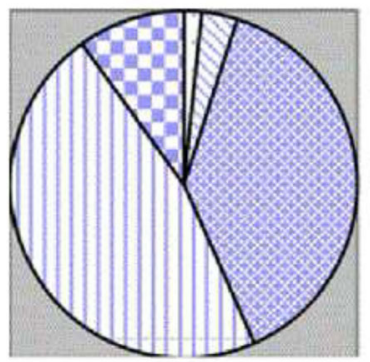

Controlled L3-4

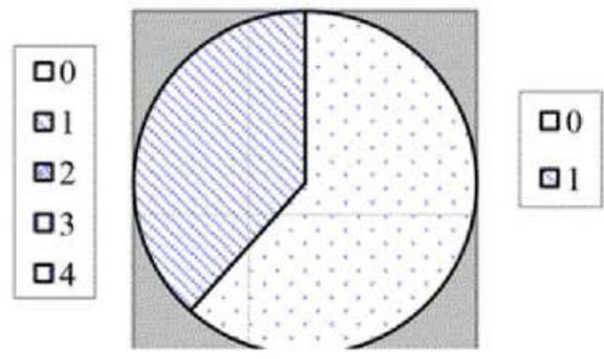

Controlled L5-S1
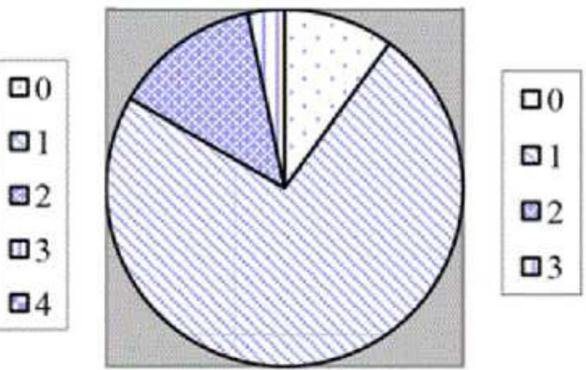

Fig. 4 Distribution of multifidus atrophy in the herniated and control groups. 0: grade 0 of multifidus; 1 : grade 1 of multifidus; 2 : grade 2 of multifidus; 3 : grade 3 of multifidus; 4: grade 4 of multifidus

the axial compression force on the disc to the anterior longitudinal ligament by switching compression loading to stretch loading; additionally, it protects the discs by preventing unexpected movements like torsion and flexion [22]. This phenomenon not only requires sufficient strength muscle, but also completes the nucleus pulposus and annulus fibrosus structure. For the L5-S1 segment, however, the intervertebral disc below the iliac crest, the ilium could restrict segment movement, thus reduce the pressure and shear forces on the intervertebral disc.

We found no correlation between L5-S1 level disc degeneration and multifidus muscle atrophy in our study. At the L3-L4 level, as there was no denervation phenomenon, the positive correlation demonstrated that LMA could be the cause of disc degeneration. At the L5-S1 level, however, the differences of LMA between the two groups suggested that LMA could also be the consequence of L4-L5 disc herniation.

Table 2 Wilcoxon rank sum test between herniated and control group lumbar multifidus atrophy

\begin{tabular}{|c|c|c|c|}
\hline & L3-L4 (control) & L4-L5 (control) & L5-S1 (control) \\
\hline $\begin{array}{l}\text { L3-L4 } \\
\text { (herniated) }\end{array}$ & $-5.126^{* *}(P<0.01)$ & & \\
\hline $\begin{array}{l}\text { L4-L5 } \\
\text { (herniated) }\end{array}$ & & $-5.818^{* *}(P<0.01)$ & \\
\hline $\begin{array}{l}\text { L5-S1 } \\
\text { (herniated) }\end{array}$ & & & $-6.217^{* *}(P<0.01)$ \\
\hline
\end{tabular}

This study analyzed the lumbar degenerative changes between herniated and adjacent segments in L4-L5 herniated disc patients using MRI. The multifidus muscle plays a critical role in controlling and stabilizing the lumbar spine in multiple planes of action, including axial rotation as well as posterior rotation (extension) on the sagittal plane [23].

Lower back pain can recollapse more often in case of weakened paraspinal muscles resulting in instability; the latter can be improved by rehabilitation exercise programs $[24,25]$. For disc-herniated patients, microdiscectomy results in minimal atrophy and fatty infiltration to the multifidus muscle [26-29]. And lumbar extension muscle-strengthening program can decrease paraspinal muscle atrophy after lumbar disc herniation surgery, and

Table 3 Correlation analysis of lumbar multifidus atrophy and disc degeneration by Spearman's rho test

\begin{tabular}{lllllll}
\hline & L3-L4 & L3-L4 & L4-L5 & L4-L5 & L5-S1 & L5-S1 \\
& LDD & LMA & LDD & LMA & LDD & LMA \\
\hline L3-L4 LDD & 1.000 & $0.352^{* *}$ & 0.125 & 0.133 & $0.283^{*}$ & 0.124 \\
L3-L4 LMA & & 1.000 & 0.243 & $0.591^{* *}$ & 0.009 & $0.439^{* *}$ \\
L4-L5 LDD & & & 1.000 & 0.058 & 0.097 & 0.006 \\
L4-L5 LMA & & & & 1.000 & -0.094 & $0.872^{* *}$ \\
L5-S1 LDD & & & & & 1.000 & -0.036 \\
L5-S1 LMA & & & & & & 1.000 \\
\hline
\end{tabular}

$L M A$ lumbar multifidus atrophy, $L D D$ lumbar disc degeneration ${ }^{*} P<0.05 ;{ }^{* *} P<0.01$ 
Table 4 Wilcoxon rank sum test between L3-4 and L5-S1 lumbar disc degeneration changes

\begin{tabular}{llll}
\hline & L3-L4 LMA & L3-L4 facet joints & L3-L4 LDD \\
\hline L5-S1 LMA & $-6.481^{* *}(P<0.01)$ & - & - \\
L5-S1 facet & - & $-1.143(P=0.253)$ & - \\
joints & & - & $-1.119(P=0.263)$ \\
L5-S1 LDD & - & - & -
\end{tabular}

$L M A$ lumbar multifidus atrophy, $L D D$ lumbar disc degeneration ${ }^{* *} P<0.01$

prevent adjacent level degeneration and lumbar spondylolisthesis, especially for patients with severe LMA caused by lower lumbar range of motion (ROM) after posterior lumbar interbody fusion.

Choi et al. [30] demonstrated that pain, duration of recuperation, and back-muscle strength can be affected favorably by lumbar extension muscle-strengthening programs after lumbar disc herniation surgery. Thus, it is recommended that MRI should be performed to obtain a description of LMA in disc-herniated patients, and clinicians should pay attention to LMA when instituting lumbar extension muscle strengthening programs.

There are several limitations with this study. First, we analyzed only patients with mono-segment disc herniation at L4-L5; excluding L3-L4/L5-S1 disc herniation and multi-segment degeneration due to the relatively small sample size. Second, we were not able to perform a comparative analysis to investigate the difference between the herniated cohort and control groups. Further study with a larger number of cases should be performed to obtain an age- and sex-matched control result and thus confirm the results of the present study.

\section{Conclusions}

This study analyzed the lumbar degenerative changes between herniated and adjacent segments in L4-L5 disc herniated patients. There was severe LMA in the L5-S1 segment. Our data confirmed that LMA contributes to the etiology of disc degeneration and/or disc herniation, especially at the L3-L4 level.

Muscle atrophy should be considered in the treatment of lumbar disc herniation patients due to its contribution to clinical symptoms. Lumbar extension muscle strengthening programs could be effective in preventing muscle atrophy and lumbar spinal degeneration.

\section{Abbreviations}

CSA: Cross-sectional area; LDD: Lumbar disc degeneration; LDH: Lumbar disc herniation; LMA: Lumbar multifidus atrophy; MRI: Magnetic resonance imaging; ROM: Range of motion

\section{Acknowledgments}

None.

Funding

None.
Availability of data and materials

The data supporting our findings can be found in the article.

\section{Authors' contributions \\ SD designed and performed the study, and wrote and edited the manuscript. $\mathrm{LP}$ revised the manuscript, conceived and designed the study, and analyzed and interpreted the data. CJ drafted and revised the manuscript. MZK and LJP assisted with data presentation, drafting, and revision of the manuscript. QTZ conceived and designed the study, and assisted with the research, drafting, and revision of the manuscript. All authors read and approved the final manuscript.}

\section{Competing interests}

No funds were received in support of this work. No benefits in any form have been or will be received from a commercial party related directly or indirectly to the subject of this manuscript.

Ethics approval and consent to participate

The experimental protocol was established according to the ethical guidelines of the Helsinki Declaration and was approved by the Human Ethics Committee of China-Japan Union Hospital of Jilin University. Written informed consent was obtained from all participants.

\section{Publisher's Note}

Springer Nature remains neutral with regard to jurisdictional claims in published maps and institutional affiliations.

Received: 9 May 2016 Accepted: 7 April 2017

Published online: 20 April 2017

References

1. Porter RW. Management of back pain. London: Churchill Livingstone; 1986. p. 89-103.

2. Bogduk N, Wilson AS, Tynan W. The human lumbar dorsal rami. J Anat. 1982;134(Pt 2):383-97.

3. Macintosh JE, Valencia F, Bogduk N, Munro RR. The morphology of the human lumbar multifidus. Clin Biomech (Bristol, Avon). 1986;1(4):196-204.

4. Macintosh JE, Bogduk N. The biomechanics of the lumbar multifidus. Clin Biomech (Bristol, Avon). 1986;1(4):205-13.

5. Zhao WP, Kawaguchi Y, Matsui H, Kanamori M, Kimura T. Histochemistry and morphology of the multifidus muscle in lumbar disc herniation: comparative study between diseased and normal sides. Spine (Phila Pa 1976). 2000;25(17):2191-9.

6. Yoshihara K, Shirai Y, Nakayama Y, Uesaka S. Histochemical changes in the multifidus muscle in patients with lumbar intervertebral disc herniation. Spine (Phila Pa 1976). 2001;26(6):622-6.

7. Freeman MD, Woodham MA, Woodham AW. The role of the lumbar multifidus in chronic low back pain: a review. PM R. 2010;2(2):142-6. quiz $141 \mathrm{p}$ following 167

8. Lee JC, Cha JG, Kim Y, Kim Yl, Shin BJ. Quantitative analysis of back muscle degeneration in the patients with the degenerative lumbar flat back using a digital image analysis: comparison with the normal controls. Spine (Phila Pa 1976). 2008;33(3):318-25.

9. Mengiardi B, Schmid MR, Boos N, Pfirrmann CW, Brunner F, Elfering A, Hodler J. Fat content of lumbar paraspinal muscles in patients with chronic low back pain and in asymptomatic volunteers: quantification with MR spectroscopy. Radiology. 2006;240(3):786-92.

10. Tabaraee E, Ahn J, Bohl DD, Phillips FM, Singh K. Quantification of multifidus atrophy and fatty infiltration following a minimally invasive microdiscectomy. Int J Spine Surg. 2015;9:25.

11. Takayama K, Kita T, Nakamura H, Kanematsu F, Yasunami T, Sakanaka H, Yamano Y. New predictive index for lumbar paraspinal muscle degeneration associated with aging. Spine (Phila Pa 1976). 2016;41(2):E84-90.

12. Battaglia PJ, Maeda Y, Welk A, Hough B, Kettner N. Reliability of the Goutallier classification in quantifying muscle fatty degeneration in the lumbar multifidus using magnetic resonance imaging. J Manipulative Physiol Ther. 2014;37(3):190-7.

13. Griffith JF, Wang YX, Antonio GE, Choi KC, Yu A, Ahuja AT, Leung PC. Modified Pfirrmann grading system for lumbar intervertebral disc degeneration. Spine (Phila Pa 1976). 2007;32(24):E708-12.

14. Pathria M, Sartoris DJ, Resnick D. Osteoarthritis of the facet joints: accuracy of oblique radiographic assessment. Radiology. 1987;164(1):227-30. 
15. Hodges $\mathrm{P}$, Holm AK, Hansson T, Holm S. Rapid atrophy of the lumbar multifidus follows experimental disc or nerve root injury. Spine (Phila Pa 1976). 2006;31(25):2926-33.

16. Bogduk N. Clinical anatomy of lumbar Spine and Sacrum. 3rd ed. Edinburgh: Churchill Livingston; 1997.

17. Bakou S, Cherel Y, Gabinaud B, Guigand L, Wyers M. Type-specific changes in fibre size and satellite cell activation following muscle denervation in two strains of turkey (Meleagris gallopavo). J Anat. 1996;188(Pt 3):677-91.

18. Bishop DL, Milton RL. The effects of denervation location on fiber type mix in self-reinnervated mouse soleus muscles. Exp Neurol. 1997;147(1):151-8.

19. Okada Y. Histochemical study on the atrophy of the quadriceps femoris muscle caused by knee joint injuries of rats. Hiroshima J Med Sci. 1989; 38(1):13-21.

20. Herbison GJ, Jaweed MM, Ditunno JF. Muscle atrophy in rats following denervation, casting, inflammation, and tenotomy. Arch Phys Med Rehabil. 1979;60(9):401-4.

21. Weber BR, Grob D, Dvorak J, Muntener M. Posterior surgical approach to the lumbar spine and its effect on the multifidus muscle. Spine (Phila Pa 1976). 1997;22(15):1765-72

22. Kader DF, Wardlaw D, Smith FW. Correlation between the MRI changes in the lumbar multifidus muscles and leg pain. Clin Radiol. 2000;55(2):145-9.

23. Richardson CA, Jull GA. Muscle control-pain control. What exercises would you prescribe? Man Ther. 1995;1 (1):2-10.

24. Oosterhuis T, Costa LO, Maher CG, de Vet HC, van Tulder MW, Ostelo RW. Rehabilitation after lumbar disc surgery. Cochrane Database Syst Rev. 2014; 3:Cd003007.

25. McGregor AH, Probyn K, Cro S, Dore CJ, Burton AK, Balague F, Pincus T, Fairbank J. Rehabilitation following surgery for lumbar spinal stenosis. Cochrane Database Syst Rev. 2013;12:Cd009644.

26. Min SH, Kim MH, Seo JB, Lee JY, Lee DH. The quantitative analysis of back muscle degeneration after posterior lumbar fusion: comparison of minimally invasive and conventional open surgery. Asian Spine J. 2009;3(2):89-95.

27. Bresnahan LE, Smith JS, Ogden AT, Quinn S, Cybulski GR, Simonian N, Natarajan RN, Fessler RD, Fessler RG. Assessment of paraspinal muscle crosssectional area following lumbar decompression: minimally invasive versus open approaches. J Spinal Disord Tech. 2013;1536-0652.

28. Pourtaheri S, Issa K, Lord E, Ajiboye R, Drysch A, Hwang K, Faloon M, Sinha $\mathrm{K}$, Emami A. Paraspinal muscle atrophy after lumbar spine surgery. Orthopedics. 2016;39(2):e209-e214.

29. Putzier M, Hartwig T, Hoff EK, Streitparth F, Strube P. Minimally invasive TLIF leads to increased muscle sparing of the multifidus muscle but not the longissimus muscle compared with conventional PLIF - a prospective randomized clinical trial. Spine J. 2016;16:811-9.

30. Choi G, Raiturker PP, Kim MJ, Chung DJ, Chae YS, Lee SH. The effect of early isolated lumbar extension exercise program for patients with herniated disc undergoing lumbar discectomy. Neurosurgery. 2005;57(4): 764-72. discussion 764-772.

\section{Submit your next manuscript to BioMed Central and we will help you at every step:}

- We accept pre-submission inquiries

- Our selector tool helps you to find the most relevant journal

- We provide round the clock customer support

- Convenient online submission

- Thorough peer review

- Inclusion in PubMed and all major indexing services

- Maximum visibility for your research

Submit your manuscript at www.biomedcentral.com/submit

CBiomed Central 